\title{
Contribution to the knowledge of Dermestidae (Coleoptera) deposited in Naturhistorisches Museum Wien. Part 2. Subfamily Thorictinae from H. Franz collection
}

\author{
K познанию жкуков-кожеедов (Coleoptera), хранящихся \\ в Естественноисторическом музее Вены. Часть 2 - \\ подсемейство Thorictinae из комлекщии Г. Франща
}

\author{
J. Háva \\ И. Гава
}

Department of Forest Protection and Entomology, Faculty of Forestry and Wood Sciences, Czech University of Life Sciences, Kamýcká 129, Prague 6 - Suchdol CZ-16521 Czech Republic. E-mail: jh.dermestidae@volny.cz.

Отдел защиты леса, факультет лесного хозяйства и лесоведения, Чешский университет наук о жизни, Прага, Чехия.

Key words: Taxonomy, new records, Coleoptera, Dermestidae, Thorictinae, Thorictus, Palaearctic Region, Canary Islands.

Ключевые слова: таксономия, новая находка, Coleoptera, Dermestidae, Thorictinae, Thorictus, Палеарктика, Канарские о-ва.

Abstract. The results of 283 Thorictinae specimen study from Herbert Franz collection deposited in Naturhistorisches Museum Wien are summarized. The species Thorictus gigas Wollaston, 1862 is newly recorded from the Lanzarote Island. A list of species recorded from the Canary Islands is provided.

Резюме. Переизучены 283 экз. подсемейства Thorictinae из коллекции Герберта Франца, хранящихся в Естественноисторическом музее Вены. Thorictus gigas Wollaston, 1862 впервые отмечается на острове Лансароте. Представлен список видов подсемейства, зарегистрированных на Канарских островах.

\section{Introduction}

The family Dermestidae (Coleoptera: Bostrichoidea) currently consists of 62 genera, containing about 1600 species worldwide [Háva, 2015, 2018]. During the determination of 283 specimens belonging to the family Dermestidae, subfamily Thorictinae (Coleoptera) deposited in Naturhistorisches Museum, Wien, Austria, coll. Herbert Franz, the author found 12 species; new faunistic records from the Canary Islands were acquired in the study presented here. The present article follows a preceding work [Háva, 2017].

\section{Material and methods}

Acronyms of material depositories: JHAC - Jiří Háva, Private Entomological Laboratory \& Collection, Únětice u Prahy, Prague-West, Czech Republic; NHMW - Naturhistorisches Museum, Wien, Austria.

Species are arranged in alphabetical order, the nomenclature and zoogeography follow Háva [2015].
Individual labels are indicated by a single slash (/), author's remarks and comments are enclosed in brackets $[\ldots]$.

\section{Results}

\author{
Thorictinae \\ Thorictus basalis John, 1968 \\ Figs 1-4.
}

Type material. Holotype: «[Morocco] Bine el Ouidane / Mittl. Atlas, b. Azilal, lg. H. Franz / Holotypus / «borictus basalis John, det. H. John», (NHMW).

Thorictus canariensis Wollaston, 1862

Material examined. «[Canary Isl.] Lanzarote, lg. H.Franz», [det. as Thorictus laticollis], 101 spec., J. Háva det., (97 NHMW, 4 JHAC); «[Canary Isl.] Lanzarote, Playa Blanca» / «Thorictus canariensis Woll, det.H.John» -1 spec., (NHMW); «Canary Isl.] Fuerteventura, lg. H.Franz», [det. as Thorictus laticollis] - 3 spec., J. Háva det., (NHMW); «[Canary Isl.] Fuerteventura, Mte. De Brtancuria, lg. H.Franz» / «Thorictus canariensis Woll, det. H. John» - 55 spec., (54 NHMW, 1 JHAC); «Canary Isl.] Gran Canaria, Umg. Maspalomas, lg. H. Franz» / «Thorictus canariensis Woll, det. H. John» 19 spec., (18 NHMW, 1 JHAC); «[Canary Isl.] El Hierro, Montana de los Tomillos, lg. H.Franz» / «Thorictus canariensis det. H. Franz» -3 spec. and 2 ants, J. Háva revid., (2 NHMW, 1 JHAC); «[Canary Isl.] El Hierro, Restinga» / «Kanarische Inseln, leg. H. Franz» -1 spec. and 2 ants, J. Háva det., (NHMW); «[Canary Isl.] El Hierro, Umg. Tinor, Kanar. Inseln, lg. Franz» - 1 spec., J. Háva det., (NHMW); «Canary Isl., Tenerife] Anagabebirge, Monte los Pasos» / «Kanarische Inseln, leg. H. Franz» / «Thiricutus cf. escalerai John, det. H. Franz» 1 spec., (NHMW).

\section{Thorictus franzi John, 1967}

Type material. Holotype: $\bigcirc^{7}$, «Gran Canaria, Umg. Pozo de las Nieves, lg. H. Franz» / «Typus» / «Thorictus franzi John, det. H. John», (NHMW). Paratype: [Allotype +]: the 

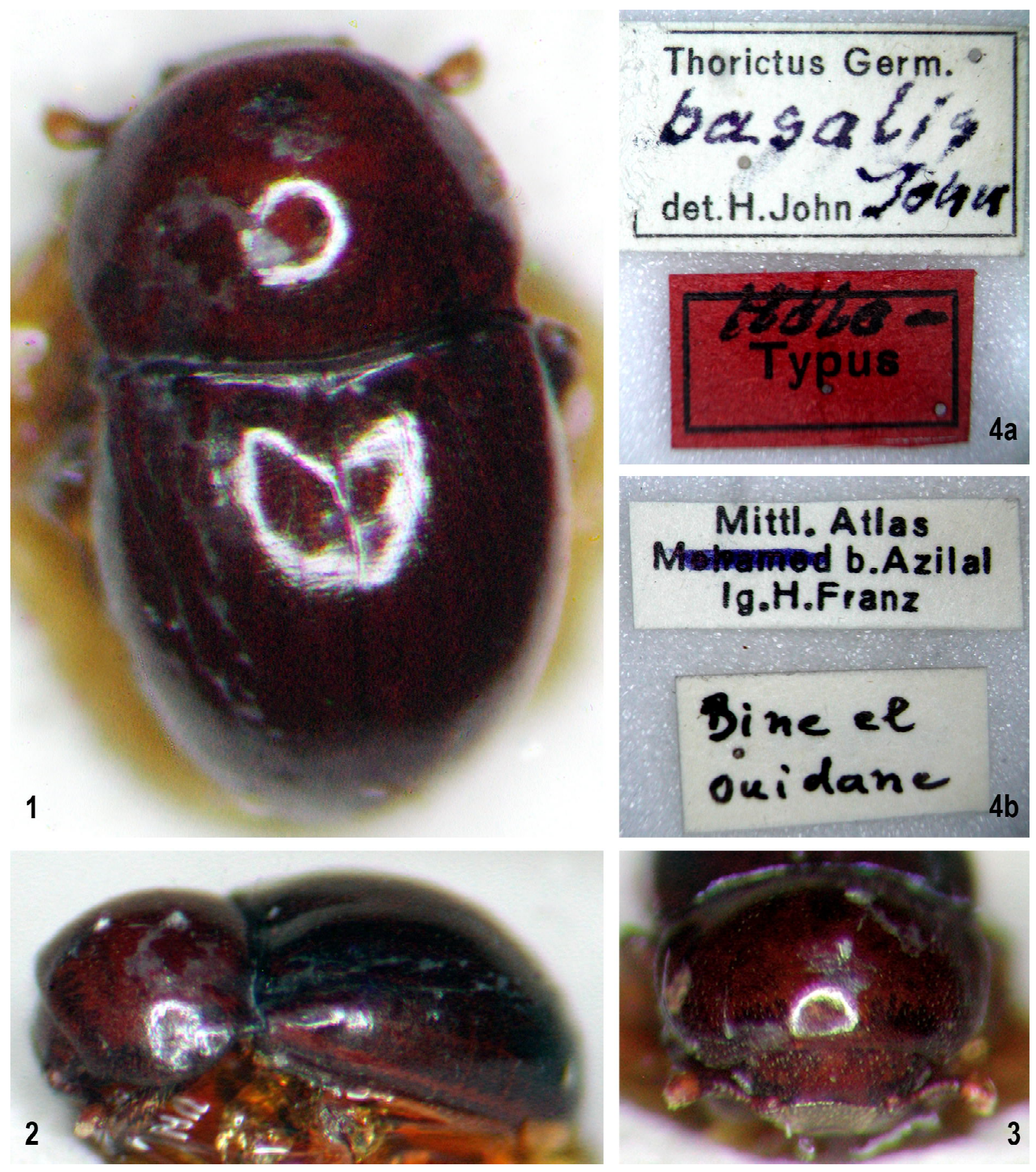

Figs 1-4. Thorictus basalis (holotype): 1 - habitus, dorsal aspect, $2-$ habitus, lateral aspect, 3 - head and pronotum, $4 \mathrm{a}-\mathrm{b}-$ original labels.

Pис. 1-4. Thorictus basalis (holotype): 1 - внешний виА, виА сверху; 2 - внешний виА, виА сбоку; 3 - голова и переднеспинка; $4 \mathrm{a}-\mathrm{b}-$ этикетки. 
same data as holotype [on the same pin as holotype], (NHMW). Paratypes: the same data as holotype (21 NHMW, 1 JHAC) 22 spec. Cotypes: the same data as holotype (19 NHMW, $1 \mathrm{JHAC})-20$ spec.

Remarks. John [1967] mentioned in original description only holotype and paratypes specimens. The specimens are erroneously labelled as «cotypes». All specimens are paratypes.

\section{Thorictus gigas Wollaston, 1862}

Material examined. «[Canary Islands] Lanzarote, entrada por los Castillos» - 1 spec., J. Háva det., (NHMW).

Remarks. The species known only from Gran Canaria, new to Lanzarote.

\section{Thorictus grandicollis grandicollis Germar, 1842}

Material examined. «[Spain] Sierra Ronda, Mte Arastepa, leg. H. Franz» / «Thorictus grandicollis Germ, det. H. John»1 spec., (NHMW); «[Spain] Umg. Sevilla, Cinca de Pino, leg. H. Franz / Thorictus loricatus Pex, det. H. Franz» -1 spec, J. Háva det., (NHMW); «[Spain] Ibiza, Umg San Miguel, lg. H. Franz / Thorictus grandicollis Germ, det. H. John» 1 spec., (NHMW); «[Spain] Sierra de Cazorla, Prov.Jaen, lg. H. Franz» / «Thorictus grandicollis Germ, det. H. John» 1 spec., (NHMW); «[Spain] Hispania, Aracena, Huelva, 19.5.57, M. González leg.» / «Thorictus grandicollis Germ, det. H. John», 1 spec., (NHMW); «[Spain] Sierra de Cazorla, Hisp. or. m.»/ «Umg. Pantano del Tranco, leg. H.Franz» / «Thorictus grandicollis Germ, det. H. John» - 2 spec., (NHMW); «[Spain] Villamartin, Guadalete» / «Thorictus grandicollis Germ, det. H. John» 1 spec., (NHMW); «[Spain] Alumecar, Andalusien» - 1 spec., J. Háva det., (NHMW); «[Spain] Menorca, Umg. Villacartos» / «Thorictus grandicollis Germ, det. H. John» - 1 spec., (NHMW); «[Spain] Sierra de Gredos, Hi. centr., lg. H. Franz» / «Thorictus grandicollis Germ, det. H. John» - 1 spec., (NHMW); «Spain] San Roque, Prov. Cadiz» / «Thorictus grandicollis Germ, det. H. John» - 2 spec., (NHMW); «[Portugal] Umg. Tavira, Algarve, S-Portugal», / «Thorictus grandicollis Germ, det. H. John»6 spec., (NHMW); «Marokko, Umg. Ounara, lg. H. Franz» «Thorictus grandicollis Germ, det. H. John» - 1 spec., (NHMW); «Nord Marokko, Umg. Xauen» / «Thorictus grandicollis Germ, det. H. John», 1 spec., (NHMW); «Ostl. Rif, Umg. Beni Bajalo» / «Thorictus grandicollis Germ, det. H. John» - 1 spec., (NHMW)

Table 1. List of Thorictus species recorded from Canary islands

Таблица 1. Список видов Thorictus, отмеченных на Канарских островах

\begin{tabular}{|c|c|c|c|c|c|c|c|}
\hline Species & $\mathrm{H}$ & $\mathrm{P}$ & G & $\mathrm{T}$ & C & $\mathrm{F}$ & $\mathrm{L}$ \\
\hline T. canariensis Wollaston, 1862 (= obsitus Wollaston, 1864) & * & * & * & * & * & * & * \\
\hline T. cobosi John et Andreae, 1967 (= cobosi John, 1969) & & & * & & & & \\
\hline T. deviedmai John, 1965 & & & & * & & & \\
\hline T. franzi John, 1967 & & & & & * & & \\
\hline T. gigas Wollaston, 1862 & & & & & * & & *! \\
\hline T. helleri John, 1963 & & & & * & & & \\
\hline T. lindbergi John, 1963 & & & & & * & & \\
\hline T. palmensis Háva \& Krásenský, 2015 & & * & & & & & \\
\hline T. subpusilus John, 1965 & & & & * & & & \\
\hline T. tamadabanus John, 1964 & & & & & * & & \\
\hline T. tejedanus John \& Andreae, 1967 (= tejedanus John, 1969) & & & & & * & & \\
\hline T. tenerifanus John, 1965 & & & & * & & & \\
\hline T. vestitus Wollaston, 1864 & & & & & & & * \\
\hline T. wollastoni John, 1963 & & & & * & * & & \\
\hline Total species & 1 & 2 & 2 & 6 & 7 & 1 & 3 \\
\hline
\end{tabular}

Abbreviations. H - El Hiero, P - La Palma, G - La Gomera, T - Tenerife, C - Gran Canaria, F - Fuerteventura, L Lanzarote. $* !-$ new record.

\section{Thorictus mauritanicus Lucas, 1846}

Material examined. «[Spain] Umg. Baza, Hi.m., lg. H. Franz» / «Thorictus mauritanicus Lec, det. H. John» 2 spec., (NHMW); «[Spain] Vaciamadrid, Hisp. c., lg. H. Franz» ( «Thorictus mauretanicus, det. H. Franz» - 1 spec., J. Háva revid., (NHMW); «Sierra, Bedmar u. Jodar» / «Thorictus mauritanicus Lec, det. H. John» - 1 spec., (NHMW); «[Morocco] Mittlerer Atlas, b. Sefrou, lg. H. Franz» / «Thorictus mauritanicus Lec, det. H. John» - 1 spec., (NHMW).

\section{Thorictus mogadoricus Escalera, 1914} Amizmiz und Marrakech, lg. H.Franz» - 1 spec., J. Háva det., (NHMW)

\section{Thorictus orientalis Peyron, 1857}

Material examined. «Turkey] Umg. Mugla, W-Anatolien, leg. H. Franz» / «Thorictus orientalis n. var., det. H. John»1 spec., J. Háva det., (NHMW).

\section{Thorictus palmensis}

Háva et Krásenský, 2015

Material examined. «[Canary Islands] La Palma, Cumbre, Roque Muchacho, lg. H. Franz» - 1 spec., J. Háva det., (NHMW); «[Canary Islands] La Palma, Cumbre über $2000 \mathrm{~m}$, lg. H. Franz» - 1 spec., J. Háva det., (JHAC).

Remarks. The species is considered based on the holotype specimen, next two known specimens and new locality data from La Palma.

\section{Thorictus stricticollis stricticollis Kraatz, 1859}

Material examined. «AAlgeria] Umg. Algier, Arbatache b. Khemis el K[h]echna» - 2 spec., J. Háva det., (NHMW, JHAC).

\section{Thorictus sulcicollis \\ Perez Arcas, 1868}

Material examined: «[Spain] Puerto de Parales, Sierra de Gata» / «Thorictus puncticollis Luc., det. H. John» - 16 spec., (14 NHMW, 2 JHAC)
Material examined. «[Morocco] Steppen zwischen 
Remarks. The mentioned specimens determined by H. John as T. puncticollis, but the species differs from sulcicollis by morphological characters. The species puncticollis is known as endemic to Algeria.

Thorictus wollastoni John, 1963

Material examined. «[Canary Islands] Gran Canaria, Umg.Maspalomas, lg. H.Franz» - 4 spec., J. Háva det., (NHMW).

\section{Acknowledgements}

I am grateful to Harald Schillhamer (NHMW) for the loan of the type material, and other specimens used in this study and to Miloslav Rakoviè (Czech Republic) for revision of the English manuscript. The research was supported by the Internal Grant Agency (B0118/004), Faculty of Forestry and Wood Sciences, Czech University of Life Sciences Prague.

\section{References}

Háva J. 2015. World Catalogue of Insects. Volume 13. Dermestidae (Coleoptera). Leiden/Boston: Brill, xxvi $+419 \mathrm{p}$.

Háva J. 2017. Contribution to the knowledge of Dermestidae (Coleoptera) deposited in Naturhistorisches Museum Wien, with desriptions of three new species // Studies and Reports, Taxonomical Series. Vol.13. No.2. P.287-297.

Háva J. 2018. World Catalogue of Dermestidae (Coleoptera). http://www.dermestidae.wz.cz. Version: February 2018. 